\title{
MOLECULAR GENETIC STUDY OF A JAPANESE FAMILY WITH LESCH-NYHAN SYNDROME: A POINT MUTATION AT THE CONSENSUS REGION OF RNA SPLICING (HPRT KEIO)
}

\author{
Yasukazu Yamada, ${ }^{1, *}$ Haruko Goto, ${ }^{1}$ Shozo TAmura, ${ }^{2}$ \\ and Nobuaki OGASAWARA ${ }^{1}$ \\ 'Department of Genetics, Institute for Developmental Research, \\ Aichi Prefectural Colony, \\ Kasugai, Aichi 480-03, Japan \\ ${ }^{2}$ Tamura Clinic, \\ Shinjuku-ku, Tokyo 160, Japan
}

\begin{abstract}
Summary Complete deficiency of hypoxanthine guanine phosphoribosyltransferase (HPRT) causes Lesch-Nyhan syndrome. A single nucleotide substitution of $\mathrm{G}$ to $\mathrm{T}$ at the $3^{\prime}$-end of intron 3 in the splicing consensus region has been identified in one allele of the HPRT gene from a mother predicted to be a heterozygous Lesch-Nyhan carrier. Utilizing a $B f a \mathrm{I}$ restriction site which was lost in the mutation as an indicator, family study showed that the mother and her only daughter were heterozygotes but the mother's sister did not have the mutant allele. The mutation generated splicing error and resulted in two types of abnormal mRNA. The major altered mRNA, named Type I, skipped the exon 4 and is predicted to produce a protein deleted of 22 amino acid residues. The other, Type II, having a 9-bp deletion at the 5 -end of exon 4 , can result in a protein lacking 3 amino acids, from codon 107 to 109 .
\end{abstract}

Key Words Lesch-Nyhan syndrome, hypoxanthine guanine phosphoribosyltransferase, sequence analysis, point mutation, altered RNA splicing

\section{INTRODUCTION}

Deficiency of a purine salvage enzyme, hypoxanthine guanine phosphoribosyltransferase [HPRT, EC 2.4.2.8], is associated with two distinct clinical disorders which are inherited as an $\mathrm{X}$ linked recessive trait. Complete deficiency of HPRT leads to Lesch-Nyhan syndrome (Seegmiller et al., 1967), whereas partial deficiency causes a severe form of gout (Kelley et al., 1967). The HPRT gene is located on

Received August 3, 1993; Accepted October 19, 1993.

*To whom correspondence should be addressed. 
the long arm of the $\mathrm{X}$ chromosome and consists of nine exons and eight introns (Patel et al., 1986). The complete sequence totalling $57 \mathrm{~kb}$ nucleotide was determined completely by Edwards et al. (1990). Transcription of this gene produces a mRNA of $1.6 \mathrm{~kb}$, which contains a protein-encoding region of 654 nucleotides (Jolly et al., 1983). The marked genetic heterogeneity of HPRT deficiency is well known. As Sculley et al. (1992) reviewed, many different mutations at the HPRT gene locus; deletions, insertions, duplications, abnormal splicing, and point mutations at different sites of the coding region from exon 1 to 9 , have been reported, including a rare case of female (Ogasawara et al., 1989). The technique of polymerase chain reaction (PCR) amplification of reverse-transcribed mRNA has recently been used to identify the molecular basis of HPRT deficiency in a number of subjects (Davidson et al., 1988, 1989, 1991; Gibbs et al., 1989, 1990; Igarashi et al., 1989; Fujimori et al., 1990, 1992; Lightfoot et al., 1992; Sculley et al., 1991; Tarle et al., 1991). Further, the multiplex amplification technique of all nine HPRT exons from the genomic DNA (Gibbs et al., 1990; Gordon et al., 1991; Yamada et al., 1992) has enabled the genomic analysis of HPRT mutations. We report here the identification of a point mutation at the consensus region for splicing of the HPRT gene in a Japanese family with Lesch-Nyhan syndrome. The mutation generated two types of abnormal mRNA because of the altered RNA splicing.

\section{MATERIALS AND METHODS}

All the methods of HPRT gene analysis, identification of the genomic mutation and the altered mRNA, were described previously (Yamada et al., 1992). Blymphoblastoid cell lines were established and maintained in RPMI 1640 medium containing 15\% FCS (Gibco Laboratories), penicillin (100 units $/ \mathrm{ml}$ ), and streptomycin $(100 \mu \mathrm{g} / \mathrm{ml})$ at $37^{\circ} \mathrm{C}$ in $5 \% \mathrm{CO}_{2}$. Selection for $\mathrm{HPRT}^{-}$cells was carried out in a medium containing $20 \mu \mathrm{M}$ 6-thioguanine (6-TG). DNA sequences were determined according to the simplified direct sequencing method described by Yamada et al. (1992), and were recorded into a personal computer and analyzed with a software of gene analysis, GENETYX version 8.0 (SDC, Japan).

\section{RESULTS AND DISCUSSION}

We investigated the molecular genetic basis of the Lesch-Nyhan syndrome in a Japanese family with the disease. The mother was predicted to be a heterozygous carrier since her two sons who were dead were both diagnosed as LeschNyhan syndrome. The genomic DNA of the mother was first analyzed. The multiplex amplification from genomic DNA revealed no differences in product sizes between the mother and normal control (data not shown). By direct sequencing of all nine amplified exons, a single nucleotide substitution of $G$ to $T$ at the 


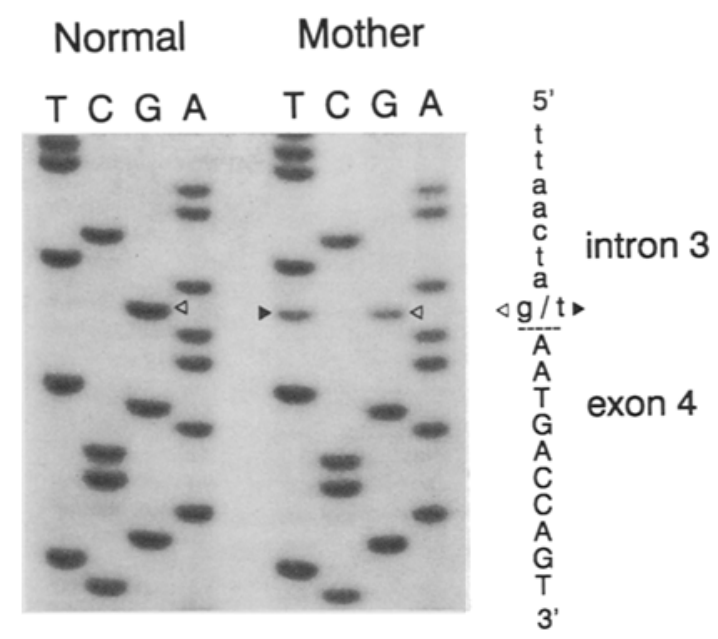

Fig. 1. Direct sequencing of the HPRT genomic DNA from a normal subject and the mother predicted to be a heterozygous Lesch-Nyhan carrier. The genomic DNA fragments were sequenced using an antisense primer HCB5 (5'CTTTCCAGTTAAAGTTGAGA3 ).

3 -end of intron 3 has been identified in one allele of the HPRT gene (Fig. 1). The mutation should result in splicing error due to the alteration of the splice acceptor site, since the $5^{\prime}$-splice consensus sequence AG was changed to AT.

The $B f a$ I restriction site (CATG) at the $3^{\prime}$-end of intron 3 is lost in the mutant allele (CTAT). Family study was performed by the PCR amplification of the exon 4 fragment and digestion by $B f a I$ (Fig. 2). DNA fragments of 334 bp including exon 4 and its franking intron sequences were amplified from the genomic DNAs of the mother, her daughter, the mother's sister, and a normal control female. The fragment from the normal allele was digested to 34,98 , and 202 bp by two $B f a$ I sites, but that from the mutant allele to 34 and $300 \mathrm{bp}$ by only one $B f a \mathrm{I}$ site. In Fig. 2 , the 34-bp band was not detected in electrophoresis because of its small size. The analysis of the mother's and her daughter's samples showed three DNA bands, the $300 \mathrm{bp}$ band from the mutant allele and the two bands, 98 and $202 \mathrm{bp}$, from the normal allele. The sequence analysis of the DNA fragment of the daughter showed both $G$ and $T$ bands at the mutation site (data not shown), as seen in the DNA sequence analysis of the mother (Fig. 1). The mother's sister had two normal a lleles. Prenatal diagnosis in this family can be performed easily if genomic DNA is isolated from chorionic villus samples (CVS), as carried out previously in another family (Yamada et al., 1992).

To clarify the alteration of mRNA generated by the identified mutation, we analyzed the HPRT cDNA amplified by RT-PCR. Amplification of the entire coding region or portions of HPRT cDNA from random B-lymphoblasts derived from the mother's blood cell resulted in same-sized DNA fragments as a normal 


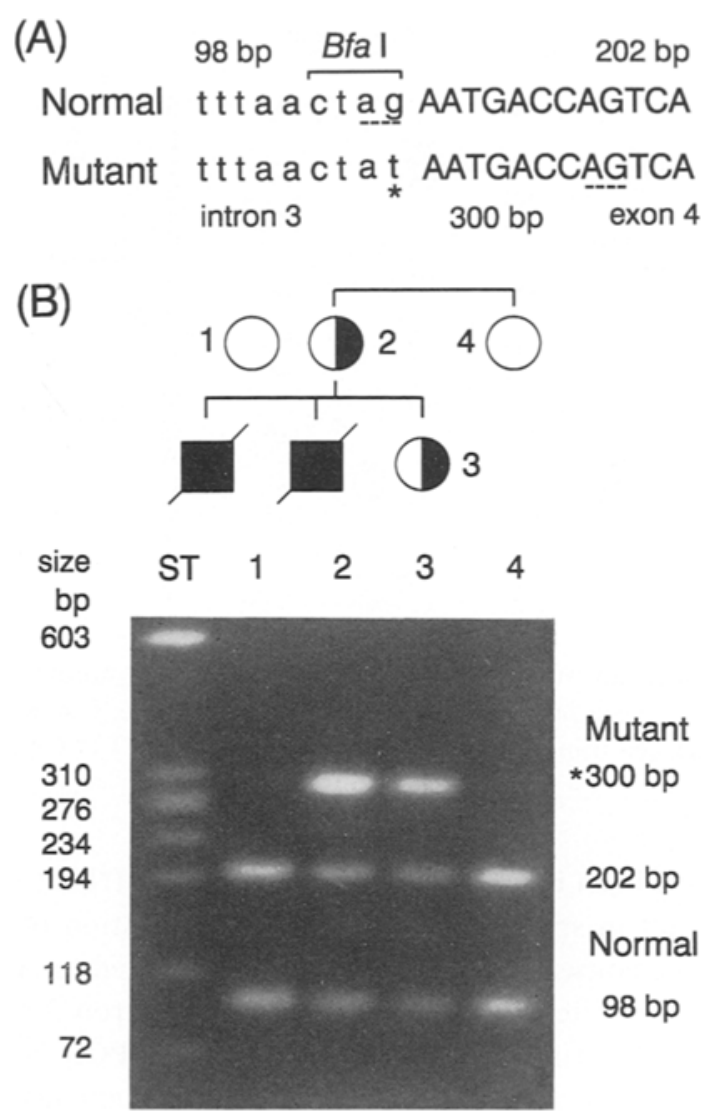

Fig. 2. Family study to detect the mutant allele. (A) The alteration of the HPRT genomic DNA. (B) Agarose gel electrophoresis (3.0\% NuSieve 3:1 agarose). The 334-bp DNA fragments were amplified using a sense primer HGE4A (5'-TAGCTAGCTAACTTCTCAAATCTTCTAG-3', on the intron 3) and an antisense primer HGE4B (5'-ATTACCTAGACTGCTTCCAAGGG-3', intron 4), and digested by Bfal. ST, DNA size marker ( $\phi$ X174/HaeIII digest); 1 , normal female; 2 , the mother; 3 , her daughter; 4 , mother's sister.

subject (data not shown). Direct sequencing of the DNA fragments or sequencing of the cloned recombinants could not detect any alterations. Most transcripts seemed to be normal. Therefore, HPRT ${ }^{-}$cells were selected from the mother's B-lymphoblasts in the presence of 6-TG. The cDNA amplification from the HP$\mathrm{RT}^{-}$cells are shown in Fig. 3A. The amplification from the HPRT- cells using the primers, HCA2 on the exon 3 and HCB3 on the exon $6 / 7$ junction resulted in a main fragment (M23) which was much shorter than the normal fragment (N23, $348 \mathrm{bp}$ ), and small amounts of fragment close to normal size. Further, the fragment (M25) amplified from the HPRT- cells by HCA2 and HCB5 on the exon 4 was slightly shorter than that from normal cells (N25, 238 bp). Direct sequencing 


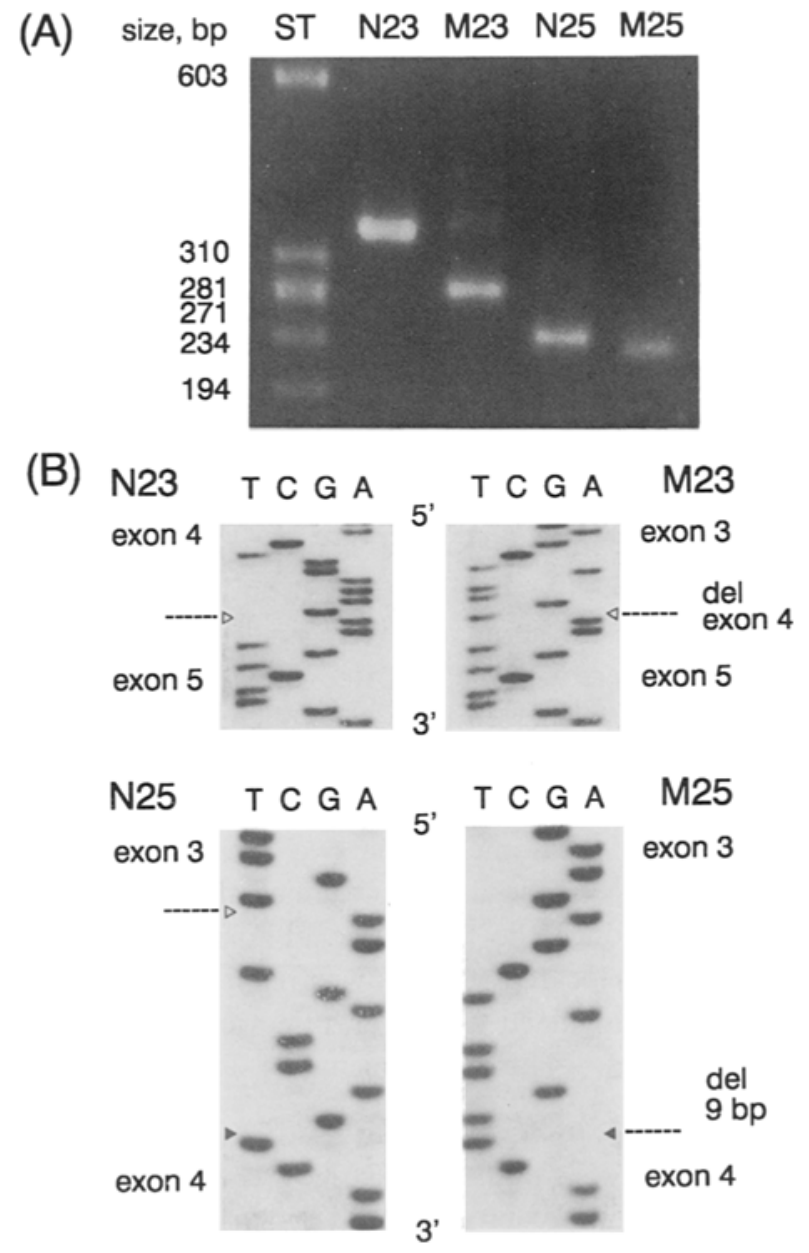

Fig. 3. Analysis of HPRT cDNA from the mother's HPRT ${ }^{-}$cells. (A) Agarose gel electrophoresis $(3.0 \%$ NuSieve $3: 1$ agarose) of PCR product from normal and the mutant cell. The cDNA fragments N23 (normal) and M23 (mutant) were amplified using a sense primer HCA2 (5'-TGCTCGAGATGTGATGAAGG-3', on the exon 3) and an antisense primer HCB3 (5'-ACCAGCAAGCTTGCGACCTT-3', exon 6/7). N25 (normal) and M25 (mutant) were amplified using the primers HCA2 and HCB5 (5'-CTTTCCAGTTAAAGTTGAGA-3', exon 4). $\phi \mathrm{X} 174 /$ Hae III digest was used as standard DNA size markers (ST). (B) Direct sequencing of the PCR products from cDNA. The fragments N23 and M23 were sequenced directly using the antisense primer HCB3, and N25 and M25 using HCB5.

of these cDNA fragments demonstrated that M23 had a deletion of all 66 bp of the exon 4 and M25 lost 9 bp at the $5^{\prime}$-end of exon 4 (Fig. 3B). Thus, there are 
two types of abnormal mRNA: Type I which is the major altered mRNA skipped the exon 4, and Type II revealed a 9-bp deletion at the 5'-end of exon 4. In Type $\mathbb{I}$, exon 4 might be skipped because of splicing error of the primary transcript due to a mutated 5'-splice site of exon 4. And, Type II was probably spliced by recognizing AG:TC 8-11 bp downstream from the mutation site (Fig. 2A). Type I mRNA results in a protein deleted of the 22 amino acid residues of exon 4 and Type II produces a protein lacking 3 amino acids, Asn-Asp-Gln, from codon 107 to 109 .

Five cases of point mutation at the splice sites in introns that may lead to altered HPRT splice patterns were reported (Gibbs et al., 1990; Gordon et al., 1991): at the splice accepter sites of exons 2 and 9, and the donor sites of exons 6,7 , and 8 . However, a new finding of a single nucleotide substitution at the accepter site of exon 4 has been identified in this study. The deletion of exon 4 has been found in 3 different families by cDNA analyses (Davidson et al, 1991; Tarle et al., 1991), but the genomic mutations in these cases were unclear. Some of them might have been caused by a splicing error. We reported previously a mutant with 4-bp deletion at the 5'-end of exon 4 generating altered splicing (Yamada et al., 1992). The mutation resulted in the formation of three different types of abnormal mRNA. One of them, type A, revealed a 4-bp deletion at the $5^{\prime}$-end of exon 4, and the other two, types B and C, were the same as the two abnormal mRNAs shown in this study, Type $I 1$ and $I$, respectively. It is presumed that mutations at the $5^{\prime}$-splice site of exon 4 always produce the latter two abnormal mRNAs. The secondary junction AG:TC which is located 9-bp downstream from the normal junction of intron 3 and exon 4 is recognized as the cryptic splice site in only mutant genes, but never in normal genes. The mechanisms of RNA splicing deserves further study.

Acknowledgments This work was supported by a Gout Research Foundation grant, an Intractable Diseases grant from the Ministry of Health and Welfare of Japan, and a grant from the Ministry of Education, Science and Culture of Japan.

\section{REFERENCES}

Davisdon BL, Chen SC, Wilson JM, Kelley WN, Palella TD (1988): Hypoxanthine guanine phosphoribosyltransferase: genetic evidence for identical mutations in two partially deficient subjects. J Clin Invest 82: 2164-2167

Davidson BL, Tarle SA, Palella TD, Kelley WN (1989): The molecular basis of HPRT deficiency in ten subjects determined by direct sequencing of amplified transcripts. J Clin Invest 84: $342-$ 346

Davidson BL, Tarle SA, Van Antwerp M, Gibbs DA, Watts RWE, Kelley WN, Palella TD (1991): Identification of 17 independent mutations responsible for human hypoxanthine-guanine phosphoribosyltransferase (HPRT) deficiency. Am J Hum Genet 48: 951-958

Edwards A, Voss H, Rice P, Civitello A, Stagemann J, Schwager C, Zimmermann J, Erfle H, Caskey CT, Ansorge W (1990): Automated DNA sequencing of the human HPRT locus. Genomics 6: $593-608$ 
Fujimori S, Kamatani N, Nishida Y, Ogasawara N, Akaoka I (1990): Hypoxanthine guanine phosphoribosyltransferase deficiency: nucleotide substitution causing Lesch-Nyhan syndrome identified for the first time among Japanese. Hum Genet 84: 483-486

Fujimori S, Tagaya T, Kamatani N, Akaoka I (1992): A germ line mutation within the coding sequence for the putative 5-phosphoribosyl-1-pyrophosphate binding site of hypoxanthineguanine phosphoribosyltransferase (HPRT) in a Lesch-Nyhan patient: missense mutations within a functionally important region probably cause disease. Hum Genet 90: 385-388

Gibbs RA, Nguyen P-N, McBride LJ, Koepf SM, Caskey CT (1989): Identification of mutations leading to the Lesch-Nyhan syndrome by automated direct DNA sequencing of in vitro amplified cDNA. Proc Natl Acad Sci USA 86: 1919-1923

Gibbs RA, Nguyen P-N, Edwards A (1990): Multiplex DNA deletion detection and exon sequencing of the hypoxanthine phosphoribosyltransferase gene in Lesch-Nyhan families. Genomics 7: $235-244$

Gordon RB, Dawson PA, Sculley DG, Emmerson BT, Caskey CT, Gibbs RA (1991): The molecular characterization of HPRTChermside and HPRTCoorparoo: two Lesch-Nyhan patients with reduced amounts of mRNA. Gene 108: 299-304

Igarashi T, Minami M, Nishida Y (1989): Molecular analysis of hypoxanthine-guanine phosphoribosyltransferase mutation in five unrelated Japanese patient. Acta Paediatr Jpn 31: 303313

Jolly DJ, Okayama H, Berg P, Esty AC, Filpula D, Bohlen P, Johnson GG, Shively JE, Hunkapillar T, Friedmann T (1983): Isolation and characterization of a full-length expressible cDNA for human hypoxanthine phosphoribosyltransferase. Proc Natl Acad Sci USA 80: 477-481

Kelley WN, Rosenbloom FM, Henderson JF, Seegmiller JE (1967): A specific enzyme defect in gout associated with overproduction of uric acid. Proc Natl Acad Sci USA 57: 1735-1739

Lightfoot T, Joshi R, Nuki G, Snyder FF (1992): The point mutation of hypoxanthine-guanine phosphoribosyltransferase (HPRTEdinburgh) and detection by ailele-specific polymerase chain reaction. Hum Genet 88: 695-696

Ogasawara N, Stout JT, Goto H, Sonta S, Matsumoto A, Caskey CT (1989): Molecular analysis of a female Lesch-Nyhan patient. J Clin Invest 84: 1024-1027

Patel PI, Framson PE, Caskey CT, Chinault CA (1986): Fine structure of the human hypoxanthine guanine phosphoribosyltransferase gene. Mol Cell Biol 6: 393-403

Sculley DG, Dawson PA, Beacham IR, Emmerson BT, Gordon RB (1991): Hypoxanthine-guanine phosphoribosyltransferase deficiency: analysis of HPRT mutation by direct sequencing and allele specific amplification. Hum Genet 87: 688-692

Sculley DG, Dawson PA, Emmerson BT, Gordon RB (1992): A review of the molecular basis of hypoxanthine-guanine phosphoribosyltransferase (HPRT) deficiency. Hum Genet 90: 195207

Seegmiller JE, Rosenbloom FM, Kelley WN (1967): Enzyme defect associated with a sex-linked human neurological disorder and excessive purine synthesis. Science 155: 1682-1684

Tarle SA, Davidson BL, Wu VC, Zidar FJ, Seegmiller JE, Kelley WN, Palella TD (1991): Determination of the mutation responsible for the Lesch-Nyhan syndrome in 17 subjects. Genomics 10: 499-501

Yamada Y, Goto H, Suzumori K, Adachi R, Ogasawara N (1992): Molecular analysis of five independent Japanese mutant genes responsible for hypoxanthine guanine phosphoribosyltransferase (HPRT) deficiency. Hum Genet 90: 379-384 